\title{
A Quantitative Analysis of Human Calling Behavior During Medical Emergency Calls
}

\author{
Xiangyu $\mathrm{Li}^{1}$, a , Wenjun Wang ${ }^{1}$, Ning Yuan ${ }^{2}$ and Haodong Lyu ${ }^{1}$ \\ ${ }^{1}$ Tianjin Key Laboratory of Advanced Networking (TANK), School of Computer Science and \\ Technology, Tianjin University, Tianjin China, 300354 ; \\ ${ }^{2}$ Department of Basic Courses, Academy of Military Transportation, PLA, Tianjin 300161. \\ aredleaf00@126.com
}

Keywords: Medical Emergency Call, Communication Pattern, Power-law Distribution, Entropy

\begin{abstract}
Human behavior is often affected by sudden emergency directly or indirectly. In this paper, we focus on two groups of people under different types of medical emergency calls. Based on the quantitative analysis of calling volume and call interval distribution before and after the emergency call, we find that during most types of emergencies, calling volume will increase after the emergency call made and maintain a high level in the next several hours. Medical emergency makes influence on call volumes, but not affects power-law distribution in human communication behavior. From studying entropy of call detail records, we found that during the medical emergency, the richness of outgoing call numbers increases and falls after that. In this paper, we do quantitative analysis of temporal patterns of medical emergency call based on the combination of medical emergency call data and call detail record data, which not only study on the characteristics of human communication behavior in emergency situations, but also provide data basis for scientific rational allocation of aid resources and effective emergency measures.
\end{abstract}

\section{Introduction}

Research of human behavior during emergencies is of great significance for dealing with emergency events, and many researches around this aspect. There is a serious need in understanding how people change their behavior when exposed to emergencies [1]. Some researchers are explored the combination of different kinds of sudden emergencies, such as jet scare, plane crash, bombing [2], crowd panics [3,4] and fires [5,6], even large-scale disasters such as earthquakes [7]. Human behavior also be compared during different kinds occasions includes emergencies and non-emergencies which can all influence of human behavior [8].

We can study such real time changes relies on the widespread use of mobile phones, which can track real-time communications records underlying social network $[9,10]$. Mobile phones can be regard as sensors during our daily life especially emergencies to study the behavior of people behavior $[2,5]$. With the help of mobile phone data, not only help refresh our understanding of information propagation [11], but also offer a new perspective on panic $[12,13]$ and emergency protocols in a data-based environment[14].

However, fewer researches have analyzed the human communication pattern during a medical emergency call made. As we know, a medical emergency call and medical emergency is usually symbiotic. With the emergency call data, we can research human behavior of emergency call makers directly and accurately. Combined with call detail record data, researches can also be pursued on another group of people who are impacted on emergency call maker.

\section{Datasets}

We used a combination of medical emergency call records data and calling detail records (CDR) data in the study. Emergency call records data includes alarm location information, alarm phone no, emergency type et al. CDR data is from a large mobile phone company in a big city in China with the 
vast majority of users. Both of the data set cover the September of the year 2014 includes over 7000 emergency calls.

We pretreatment the raw data first before our experiments. There are several types of diseases or emergencies in the emergency call records, we classified them into four groups which types are: traffic accident, trauma, cardiovascular diseases, respiratory diseases, poisoning, digestive diseases and some other kinds of diseases or emergencies. The outgoing and incoming phone number records during making telephone calls or sending messages are encrypted with SHA1, so we encrypt phone numbers in emergency call records in the same way so that phone number records in both data set can be matched.

\section{Results and Discussion}

In our work, we study human calling behavior during medical emergency calls through the network of communications during medical emergencies. We define the group of users who made the medical emergency call by population G0, and the population who were directly called or sent messages by G0 users are defined as G1 users. We focus on the impact of medical emergency calls in both G0 users and G1 users under different medical emergencies.

\section{Call Volume Change During Emergencies}

It is obvious that call volume of G0 users can be affected directly when a medical emergency call made. We do more research on the influence of G1 users and the subsequent impact after medical emergency call for both two group users. We collected outgoing calls of four typical types of medical emergency calls of G0 users and G1 users. We define the time when G0 made the medical emergency call as the emergency time 0 , and then add up outgoing call records of two groups of users 4 hours before and after emergency time. We choose the four types of emergency calls are typical medical emergency calls with a larger quantity compared with others. They are traffic accidents, cardio-cerebrovascular diseases, wounds and respiratory diseases (Fig. 1.).
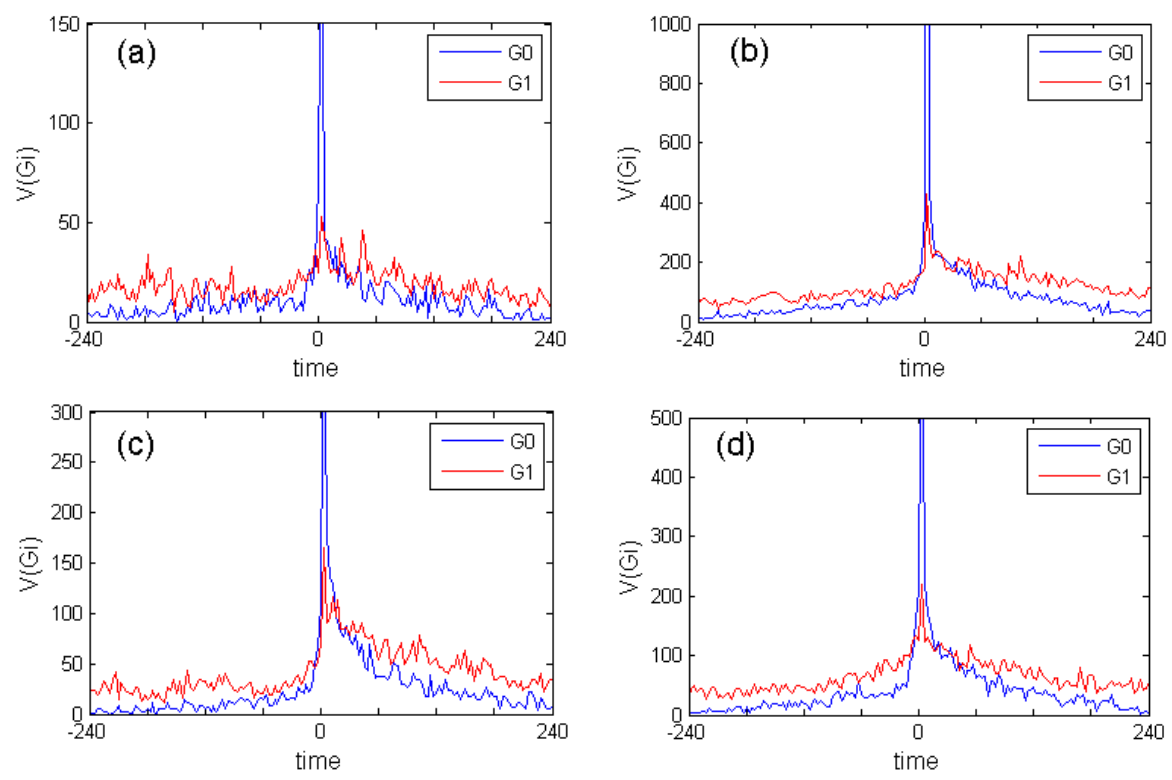

Fig. 1 Calling volume of 4 types medical emergency calls before and after the call made. Call reasons from (a) to (d) are traffic accidents, cardio-cerebrovascular diseases, wounds and respiratory diseases.

The call volume peak of G0 is overlapped so that we conceal the peak, but all G1 call volume tendency are also single peaked. In Fig. 1., we can find that at the time period G0 reached the peak, G1 call volume also reached the peak, but the total volume is much smaller than G0. G1 is a group of 
users just related with G0, so we can conclude that G1 users are often impacted by G0. We can also find that call volume after time 0 are more than before time 0 .

In medical emergency call records data, wounds and traffic accidents are classified in the same category. After calculated them respectively, there are significant differences between the tendency of Fig. 1c (traffic accidents) and Fig. 1d (wounds). G0 call volume increases 197\% after the traffic accident than before, while increases $73 \%$ after wounds. And G1 call volume increases $97 \%$ after the medical emergency call, but only increases $15 \%$ after wounds. As we all know, traffic accidents are often more serious than wounds and usually have a greater impact on the surroundings. Medical emergency calls during traffic accidents are made by eyewitnesses or the person concerned in the event, they may also lead different distribution in call volumes. In the future work, the two emergency call makers will be classified. Traffic accidents usually cause abnormal behavior of communication and influence more in human behavior than wounds.

\section{Inter-event Time Distribution of Communication Behavior}

The inter-event time distribution of human communication behavior would follow power-law distribution rather than Poisson distribution in temporal characteristics. We searched the inter-event time distribution for two groups (G0 and G1) before and after the emergency call (Fig. 2.).
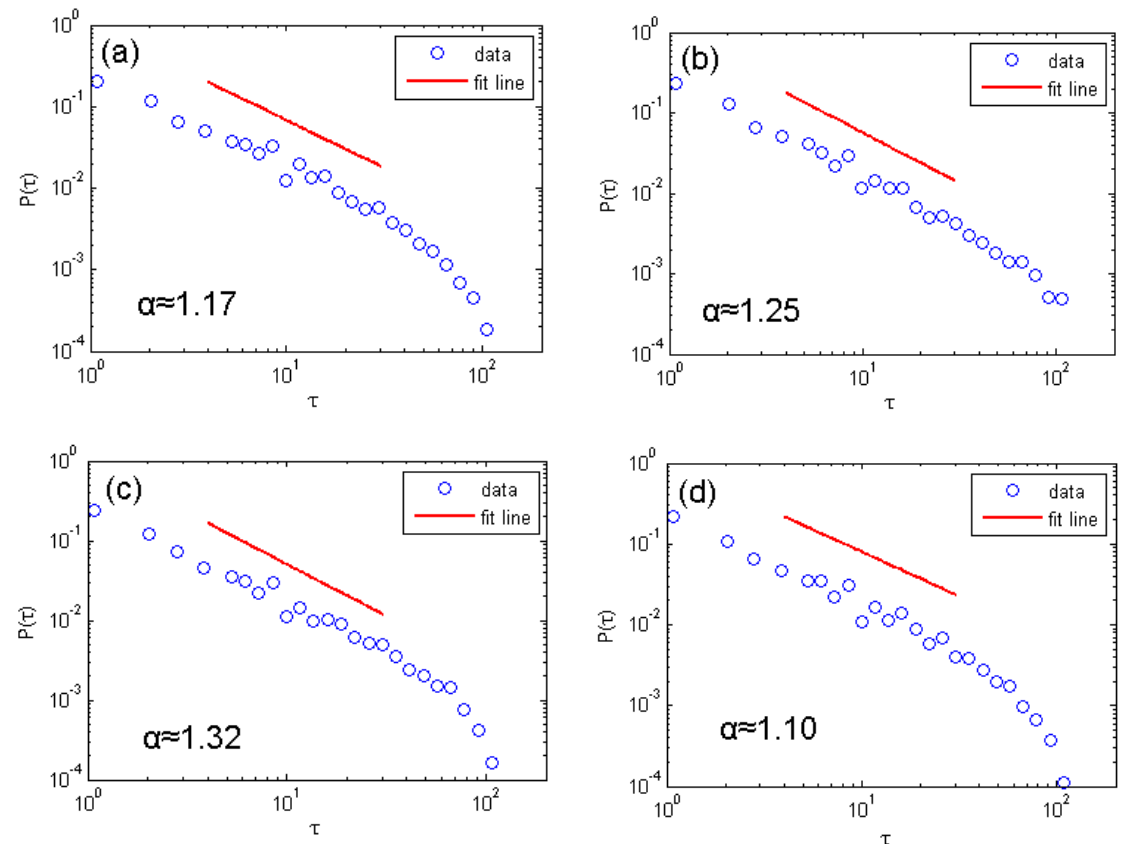

Fig. 2 Inter-event time probability density distribution for interaction between call intervals. (a) G0 before make medical emergency call. (b) G0 after make medical emergency call. (c) G1 before get medical emergency call from G0. (d) G1 after get medical emergency call.

In Fig. 2., phone call making intervals are in two hours, and inter-event time distribution fits power law distribution with slightly bulges at the interval of 10 minutes. This phenomenon is in common but not individual. Phone call volume less than four different occasions are quite different as well as before and after medical emergency call made, but the inter-event time leads almost the same distribution. We can find there is an obvious exponential decay on the tail of Fig. 2a,c,d. Inter-event time distribution in three sub figures lead power-law distribution with exponential decay while Fig. $2 \mathrm{~b}$ lead a power-law distribution and displayed straightly under log-log plot. That may be caused during some emergencies, the person who made the emergency call often doesn't have enough time to contact G1 users. 


\section{Outgoing Call Numbers Entropy Tendency During Emergencies}

As people has the home and workplace pattern, most people only have several common use phone numbers. In order to measure the richness of outgoing calls of G0 and G1 users, we calculated the entropy of outgoing calls.

Entropy is a measure of the expected value of a random variable appears, the greater the entropy of a variable, the more circumstances appears. We calculated the entropy of the phone calls made by G0 and G1 users of 3 time periods. One period contains two half hour before and after the medical emergency call made, the other two periods with forward and backward one-hour delay respectively.

$$
H=-\sum_{i=1}^{n} p_{i} \log p_{i}
$$
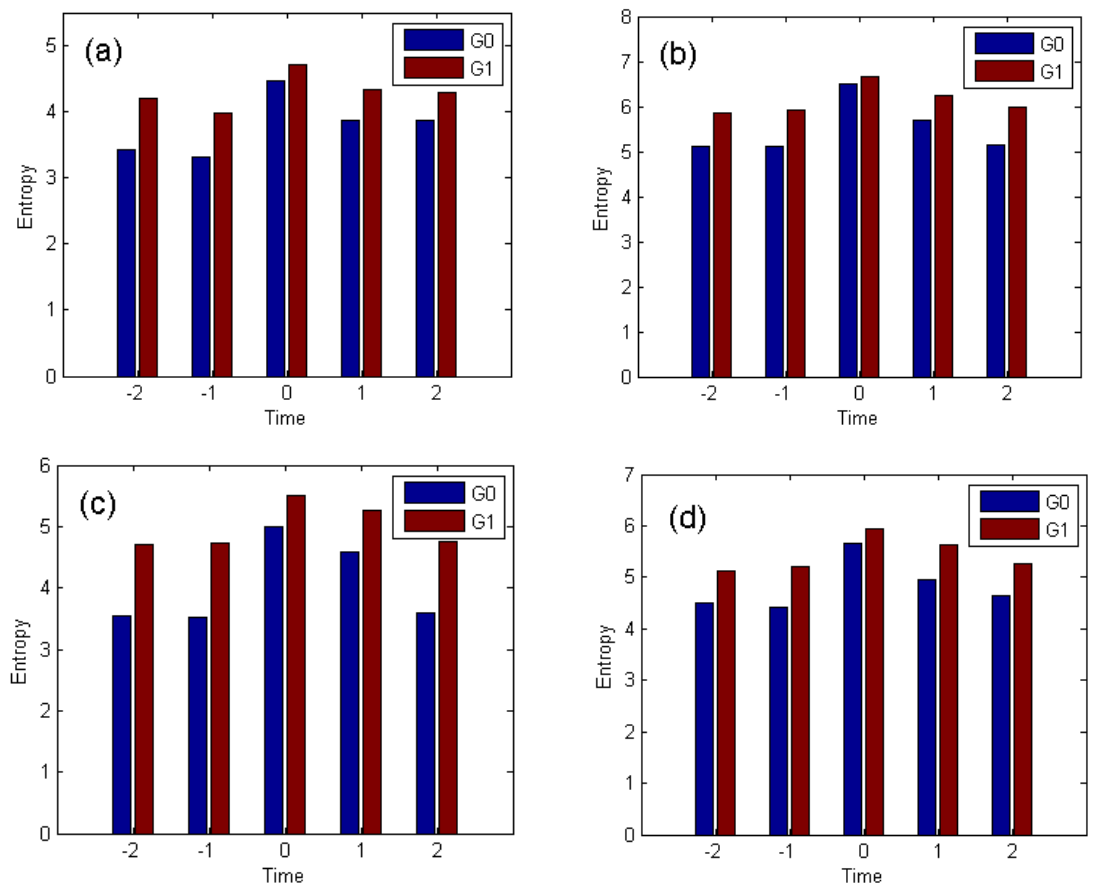

Fig. 1 Phone call number entropy tendency of four types of medical emergency calls between two groups of people (G0 and G1). From (a) to (d) are traffic accidents, cardio-cerebrovascular diseases, wounds and respiratory diseases.

From Fig. 3, we can discover tendency of call richness from G0 and G1 users. Each sub graph represents a category of medical emergency call. Time 0 indicates one hour before and after the emergency call made.

Entropy peaks of both G0 and G1 appear in the time of medical emergency call events, and usually come down to a normal level after 2 hours. From entropy tendency, we can discover that the richness of calling number usually rises in case of medical emergency. This means that people usually make more different telephone calls rather than call fixed phone numbers during emergency. We can also discover the minimum entropy gap of G0 and G1 appeared during the time of the event. It means that the entropy of G0 users rises more than G1 users during the emergency. G0 users are the eyewitnesses of the medical emergency while G1 users are impacted by G0 users.

\section{Conclusion}

In this article, we analyzed human behavior during emergency through the combination of call detail records data and medical emergency call data. From the experiment, we can conclude that call volume will surge when people in the event of a medical emergency, and this surge will spread with telephone call making. But the extent and impact in the follow-up time of the surge are different under different kinds of emergencies. There is a surge of call volume in magnitude, but human behavior still follows statistical law of human dynamics. Finally, communication behavior in 
emergency situations was explored from the perspective of entropy. It is founded that when an emergency situation occurs, G0 and G1 call entropy will both increase, which proved that people will making calls to more people under medical emergency, rather than to a few fixed people as usual. In addition, call detail record data can also be combined with other emergency calls on human behavior analysis. Quantitative analysis of calling behavior during emergency calls describes human behavior under exceptional circumstances, and it is meaningful for public safety and detection of abnormal conditions.

\section{Acknowledgement}

This work was supported by the Major Project of National Social Science Fund (14ZDB153), the major research plan of the National Natural Science Foundation (91224009, 51438009), the National Science and Technology Pillar Program (2015BAL05B00 and 2015BAL05B02).

\section{References}

[1] Vespignani A (2009) Predicting the Behavior of Techno-Social Systems. Science 325: 425-428.

[2] Gao L, Song C, Gao Z, et al. Quantifying Information Flow During Emergencies[J]. Scientific Reports, 2014: 3997-3997.

[3] Johansson A, Helbing D (2008) From crowd dynamics to crowd safety: A video-based analysis. Adv Complex Syst 11:497-527.

[4] Moussaid M, Garnier S, Theraulaz G, Helbing D (2009) Collective information processing and pattern formation in swarms, flocks, and crowds. Top Cogn Sci 1:469-497.

[5] Bickman L, McDaniel MA (1976) Model of human-behavior in fire emergencies. Bull Psychon Soc 8:254-254.

[6] Hahm J, Lee JH (2009) Human errors in evacuation behavior during a traumatic emergency using a virtual fire. Cyberpsychol Behav 12:98-98.

[7] Lu X, Bengtsson L, Holme P, et al. Predictability of population displacement after the 2010 Haiti earthquake[J]. Proceedings of the National Academy of Sciences of the United States of America, 2012, 109(29): 11576-11581.

[8] Bagrow J P, Wang D, Barabasi A, et al. Collective Response of Human Populations to Large-Scale Emergencies[J]. PLOS ONE, 2011, 6(3).

[9] Onnela JP, Sarama“ ki J, Hyvo“nen J, Szabo' G, Lazer D, et al. (2007) Structure and tie strengths in mobile communication networks. Proceedings of the National Academy of Sciences 104: $7332-7336$.

[10] Caldarelli G (2007) Scale-Free Networks. Oxford University Press.

[11] Centola D (2010) The Spread of Behavior in an Online Social Network Experiment. Science 329: $1194-1197$.

[12] Helbing D, Farkas I, Vicsek T (2000) Simulating dynamical features of escape panic. Nature 407: 487-490.

[13] Kaplan EH, Craft DL, Wein LM (2002) Emergency response to a smallpox attack: The case for mass vaccination. Proceedings of the National Academy of Sciences of the United States of America 99: 10935-10940.

[14] Kapoor A, Eagle N, Horvitz E (2010) People, quakes, and communications: Inferences from call dynamics about a seismic event and its influences on a population. In: Proceedings of AAAI Artificial Intelligence for Development (AID'

10). pp 51-56. 